\title{
Clinical and genomic factors associated with seizures in meningiomas
}

\author{
*Trisha P. Gupte, BS, ${ }^{1,2}$ Chang Li, BS,1-4 Lan Jin, PhD, ${ }^{1,2,5,6}$ Kanat Yalcin, MD,1,2 \\ Mark W. Youngblood, MD, PhD, ${ }^{7}$ Danielle F. Miyagishima, BA, ${ }^{1,2}$ Ketu Mishra-Gorur, PhD, ${ }^{1,2}$ \\ Amy Y. Zhao, BS, ${ }^{1,2}$ Joseph Antonios, MD, PhD, ${ }^{1,2}$ Anita Huttner, MD, ${ }^{2,8}$ Declan McGuone, MD, 2,8 \\ Nicholas A. Blondin, MD, ${ }^{2,9}$ Joseph N. Contessa, MD, PhD, ${ }^{2,10}$ Yawei Zhang, PhD, 5,6 \\ Robert K. Fulbright, MD, ${ }^{2,11}$ Murat Gunel, MD, ${ }^{1,2,12}$ Zeynep Erson-Omay, PhD, ${ }^{1,2}$ and \\ Jennifer Moliterno, MD ${ }^{1,2}$
}

Departments of ${ }^{1}$ Neurosurgery, ${ }^{5}$ Surgery, ${ }^{8}$ Pathology, ${ }^{9} \mathrm{Clinical}$ Neurology, ${ }^{10}$ Therapeutic Radiology and Pharmacology, ${ }^{11}$ Radiology and Biomedical Imaging, and ${ }^{12}$ Genetics, Yale School of Medicine, New Haven; ${ }^{2}$ Yale Brain Tumor Center, Smilow Cancer Hospital, New Haven, Connecticut; ' ${ }^{2}$ epertment of Neurosurgery, Xiangya Hospital, Central South University, Changsha; ${ }^{4}$ The Third Xiangya Hospital, Central South University, Changsha, Hunan, People's Republic of China; ${ }^{6}$ Department of Environmental Health Sciences, Yale School of Public Health, New Haven, Connecticut; and 'Department of Neurological Surgery, Northwestern University, Chicago, Illinois

\begin{abstract}
OBJECTIVE The association of seizures with meningiomas is poorly understood. Moreover, any relationship between seizures and the underlying meningioma genomic subgroup has not been studied. Herein, the authors report on their experience with identifying clinical and genomic factors associated with preoperative and postoperative seizure presentation in meningioma patients.
\end{abstract}

METHODS Clinical and genomic sequencing data on 394 patients surgically treated for meningioma at Yale New Haven Hospital were reviewed. Correlations between clinical, histological, or genomic variables and the occurrence of preoperative and postoperative seizures were analyzed. Logistic regression models were developed for assessing multiple risk factors for pre- and postoperative seizures. Mediation analyses were also conducted to investigate the causal pathways between genomic subgroups and seizures.

RESULTS Seventeen percent of the cohort had presented with preoperative seizures. In a univariate analysis, patients with preoperative seizures were more likely to have tumors with a somatic NF2 mutation $(p=0.020)$, WHO grade II or III tumor $(p=0.029)$, atypical histology $(p=0.004)$, edema $(p<0.001)$, brain invasion $(p=0.009)$, and worse progressionfree survival (HR 2.68, 95\% Cl 1.30-5.50). In a multivariate analysis, edema (OR 3.11,95\% Cl 1.46-6.65, $p=0.003$ ) and atypical histology $(\mathrm{OR} 2.00,95 \% \mathrm{Cl} 1.03-3.90, p=0.041)$ were positive predictors of preoperative seizures, while genomic subgroup was not, such that the effect of an NF2 mutation was indirectly mediated through atypical histology and edema $(p=0.012)$. Seizure freedom was achieved in $83.3 \%$ of the cohort, and only $20.8 \%$ of the seizure-free patients, who were more likely to have undergone gross-total resection $(p=0.031)$, were able to discontinue antiepileptic drug use postoperatively. Preoperative seizures (OR 3.54, 95\% Cl 1.37-9.12, $p=0.009$ ), recurrent tumors (OR 2.89, $95 \% \mathrm{Cl} 1.08-7.74, p=0.035)$, and tumors requiring postoperative radiation (OR 2.82, 95\% Cl 1.09-7.33, $p=0.033$ ) were significant predictors of postoperative seizures in a multivariate analysis.

CONCLUSIONS Seizures are relatively common at meningioma presentation. While NF2-mutated tumors are significantly associated with preoperative seizures, the association appears to be mediated through edema and atypical histology. Patients who undergo radiation and/or have a recurrence are at risk for postoperative seizures, regardless of the extent of resection. Preoperative seizures may indeed portend a more potentially aggressive molecular entity and challenging clinical course with a higher risk of recurrence.

https://thejns.org/doi/abs/10.3171/2020.7.JNS201042

KEYWORDS meningiomas; seizures; genomics; oncology

ABBREVIATIONS AED = antiepileptic drug; CNV = copy number variation; EOR = extent of resection; GTR = gross-total resection; $L O H=$ loss of heterozygosity; PFS = progression-free survival; PTBE = peritumoral brain edema; STR = subtotal resection; WES = whole exome sequencing.

SUBMITTED April 1, 2020. ACCEPTED July 7, 2020.

INCLUDE WHEN CITING Published online December 4, 2020; DOI: 10.3171/2020.7.JNS201042.

${ }^{*}$ T.P.G. and C.L. contributed equally to this work. 
$\mathrm{M}$ ENINGIOMAS are the most common central nervous system tumors, and approximately $30 \%-40 \%$ of patients with meningioma have seizures. ${ }^{1,2}$ Uncontrolled seizures can significantly affect the quality of life of patients with brain tumors and result in cognitive deterioration. ${ }^{3-6}$ While seizure freedom can be achieved in approximately $80 \%$ of meningioma patients after surgery, some can experience a recurrence, and nearly $20 \%$ of meningioma patients without preoperative seizures can develop seizures postoperatively. ${ }^{3,5,7,8}$ Given that worse patient outcomes have been associated with the presence of preoperative seizures, understanding the associated risk factors is critical in treating patients. ${ }^{3-5}$

Several factors related to seizure formation in patients with meningioma have been proposed. The risk of seizures is associated with tumor location, as seizures tend to be more common with supratentorial tumors that have cortical proximity than with lesions with posterior fossa or skull base locations. ${ }^{7,-12}$ The WHO grade has been identified as an independent predictor of preoperative seizures in meningiomas, and in grade II or III tumors, brain invasion appears to play an important role in seizure formation. ${ }^{13}$ Similarly, peritumoral brain edema (PTBE) has also been shown to potentially contribute to seizures in meningiomas and may be related to persistent seizures after resection..$^{5,13}$ Potentially distinct PTBE of brain-invasive meningiomas has been recently studied in particular, as cortical invasion and subsequent corresponding edema may facilitate seizures in these tumors. ${ }^{13,14}$ While genetic features such as isocitrate dehydrogenase 1 (IDH1) and 2 (IDH2) mutations have been suggested as pertinent risk factors for seizures in low-grade gliomas, $3,5,15,16$ the genomic background of meningiomas has not been correlated with seizure risk.

To better elicit the potential underlying mechanism and the correlation between genetic drivers and seizures in meningiomas, we retrospectively reviewed a large series of meningiomas and here describe the clinical and genomic correlations.

\section{Methods \\ Clinical Data Acquisition}

Electronic medical records for patients with meningiomas who had undergone resection with targeted or whole exome sequencing (WES) at the Yale New Haven Hospital and Yale Brain Tumor Center were retrospectively reviewed. The cohort included a consecutive series of patients, age 18 years and older, with a tumor histologically confirmed as a meningioma for which genomic sequencing had been performed. Patient demographics, treatment history, and tumor-specific characteristics were recorded and are described below.

Tumor Pathology: WHO Grade, Histological Subtype, Ki-67, and Brain Invasion

The histological subtype and WHO grade for all tumors were determined by board-certified neuropathologists in accordance with WHO guidelines. Ki-67 expression was recorded from available pathology reports and classified as $<5 \%$ (low) or $\geq 5 \%$ (high) with cutoffs based on findings previously relating the Ki-67 index to meningioma recurrence. ${ }^{17}$ The presence of brain invasion was also recorded from available pathology reports. After 2016, a neuropathologist retrospectively reviewed cases with brain invasion and changed the previously assigned WHO grade to grade II if brain invasion was present.

\section{Tumor Volume}

Preoperative MRI or CT imaging in all available cases was utilized to record the maximal coronal (a), axial (b), and sagittal (c) diameters of the tumor. Tumor volume was calculated using the ellipsoid formula: $(4 / 3) \pi \times(a / 2) \times$ $(\mathrm{b} / 2) \times(\mathrm{c} / 2)$.

\section{Tumor Location}

Tumor location was assessed by board-certified neuroradiologists based on preoperative MRI or CT imaging or by descriptions in operative notes when imaging was not available. We included only those tumors in which location could be determined with complete confidence. Though infratentorial meningiomas would not likely be associated with seizures, all consecutive meningiomas were included in our analysis. Tumor location was classified as "skull base" or "non-skull base," "midline" or "non-midline," and "anterior" or "posterior."

\section{Peritumoral Brain Edema}

PTBE was assessed using preoperative FLAIR MRI sequences or T2-weighted sequences when FLAIR sequences were not available. The presence of PTBE was recorded as "yes" or "no" and was used in all analyses. The extent of PTBE was further delineated on a graded scale as not present ("N"), smaller than the tumor's estimated volume ("S"), the same size as the tumor's estimated volume ("T"), or larger than the tumor's volume ("L").

\section{Extent of Resection}

Extent of resection (EOR) was determined from the operative reports, as well as with postoperative radiographic confirmation. EOR was characterized using the Simpson grading scale such that resections with Simpson grades I, II, or III were classified as gross-total resection (GTR) and resections with Simpson grade IV were classified as subtotal resection (STR). There were no biopsies in our series.

\section{Seizures and Antiepileptic Drug Use}

Charts were reviewed for seizure history, and patients were considered to have had pre- and postoperative seizures if the occurrence of seizures was temporally related to diagnosis and surgery, respectively, and believed to be a result of the tumor. Antiepileptic drug (AED) use, type of medications administered, and whether patients were taking AEDs at the time of data collection was documented. Perioperative AED administration, defined as loading the patient with an AED, usually levetiracetam (Keppra), at the time of surgery and continuing peri- and postoperatively for 10-14 days was recorded. Seizure freedom, defined as the absence of seizure, was assessed from the time of surgery until the time of data collection. While seizure freedom was considered regardless of AED use, 
we documented whether seizure freedom was in the setting of discontinuation of AEDs.

\section{Genomic Characterization of Meningiomas}

IRB approval from Yale University for this study's methods, along with informed consent from all of the included patients, was obtained. Samples were submitted for WES or targeted sequencing to identify known genomic drivers. The methods involving the targeted sequencing and annotation of genomic subgroups have been previously reported..$^{18,19}$ Using these methods, we classified genomic subgroups based on somatic alterations in the following genes: 1) NF2 (and/or Chr22q loss); 2) SMARCB1 with or without $N F 2 / \mathrm{Chr} 22$; 3) $K L F 4^{K 409 Q}$ with or without mutant TRAF7; 4) PI3K signaling, with or without mutant TRAF7; 5) Hedgehog signaling; 6) POLR2A $A^{Q 403 K}$ or

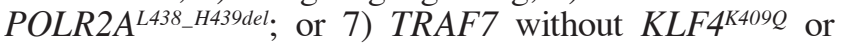
PI3K signaling mutations (Fig. 1A). All other samples were classified as "mutation unknown" and were excluded from analyses involving genomic variables. These groups herein will be referred to as "NF2," "SMARCB1," "KLF4," "PI3K," "HH," "POLR2A," and "TRAF7-alone," respectively.

For comparing mutation count load and percent of genome alteration by copy number variations (CNVs) or loss of heterozygosity (LOH), a total of 270 samples, 47 (17.4\%) from patients with preoperative seizures and 223 (82.6\%) from patients with no preoperative seizures, were analyzed.

\section{Statistical Analysis}

Statistical analysis was performed using standard statistical software (IBM SPSS Statistics, version 24, IBM Corp.). All analyses were reaffirmed by an independent statistician. Univariate analyses were performed to test clinical, histological, and genomic correlations with preand postoperative seizures. Fisher's exact test and the 1-way ANOVA test were used for categorical and continuous variables, respectively. For multivariate analyses, logistic regression models were developed, and backward stepwise model selection (by the Akaike information criterion) was performed to select final predictors from all variables that were significantly associated with pre- and postoperative seizures in univariate analyses. To visually depict the survival and log-rank statistic, the KaplanMeier survival analysis (log-rank test) was used. The Cox proportional-hazards model was used to examine associations between pre- and postoperative seizures with progression-free survival (PFS). To compare the mutation count and percent of genome alteration by $\mathrm{CNV} / \mathrm{LOH}$, the Welch two-sample t-test was conducted.

To better understand relevant factors potentially associated with the occurrence of preoperative seizures, a series of univariate analyses were conducted to test relationships between hypothesized variables. Next, mediation analyses were performed to investigate the causal impacts of certain genomic subgroups on preoperative seizures. Serial multiple mediator models were utilized to investigate the direct and indirect pathways with atypical histology, edema, and brain invasion as mediators. These models were developed using the lavaan package in $\mathrm{R}$ ( $\mathrm{R}$ Foundation for Statistical Computing). All $\mathrm{p}$ values $\leq 0.05$ were considered statistically significant.

\section{Results}

\section{Patient Characteristics}

Of the 394 patients who had appropriate data for the aforementioned variables and were included in this study, 268 were female $(68.0 \%)$, and the overall median age at diagnosis was 58 years (Fig. 1B). Median follow-up time was 21.12 months (range 15.48-26.88 months). Sixty-six patients $(16.8 \%)$ presented with preoperative seizures, and 24 patients $(6.1 \%)$ experienced postoperative seizures. Ultimately, seizure freedom was achieved in $83.3 \%$ of the preoperative seizure cohort. Eleven patients with preoperative seizures continued to exhibit postoperative seizures.

\section{Role of Prophylactic AED Use in Pre- and Postoperative Seizures}

Of the 63 patients with preoperative seizures and available information on AED usage, all were managed with AEDs preoperatively, as expected (Table 1). At the time of data collection, $79.2 \%$ of the patients in whom seizure freedom had been achieved were still actively taking AEDs. The remaining 20.8\% who were able to discontinue AED use postoperatively were more likely to have undergone a GTR ( $\mathrm{p}=0.031$ ), with the majority having WHO grade I tumors $(90.9 \%)$ and primary tumors $(81.8 \%)$. Perioperative AED administration was used in $43.7 \%$ of the patients without preoperative seizures. Of the 24 patients with postoperative seizures, 11 had received AEDs preoperatively when they had presented with preoperative seizures. The remaining 13 patients all received AEDs perioperatively; despite this, they had postoperative seizures. At the time of data collection for this study, $95.8 \%$ of postoperative seizure patients were still actively taking AEDs.

\section{Factors Associated With Preoperative Seizures}

The results from univariate analyses on associations between clinical or histological parameters and preoperative seizures are summarized in Table 2. Patients with preoperative seizures were more likely to be male $(\mathrm{p}=$ $0.014)$ and have tumors with WHO grade II or III ( $\mathrm{p}=$ $0.029)$ in a non-skull base location $(\mathrm{p}=0.012)$. They were also more likely to have tumors with PTBE $(\mathrm{p}<0.001)$, brain invasion $(\mathrm{p}=0.009)$, elevated $\mathrm{Ki}-67$ expression (i.e., $\geq 5 \%$; $=0.012)$, atypical histology $(\mathrm{p}=0.004)$, and a somatic NF2 driver mutation $(p=0.020)$. NF2 was the only genomic subgroup significantly associated with preoperative seizures across all tumor grades, whereas among WHO grade I meningiomas only, no genomic subgroups were significantly associated with preoperative seizures (Fig. 2A and C, Supplemental Tables 1-2). In further analysis, there was no statistically significant difference in the mutation count or percent of genome alteration by $\mathrm{LOH}$ events in cases with preoperative seizures compared to those without. Comparison of the percent of genome alteration by $\mathrm{CNV}$ events revealed no significance $(\mathrm{p}=$ 0.073 ), although samples from patients with preoperative seizures had CNV events in $13.0 \%$ of their genomes, 


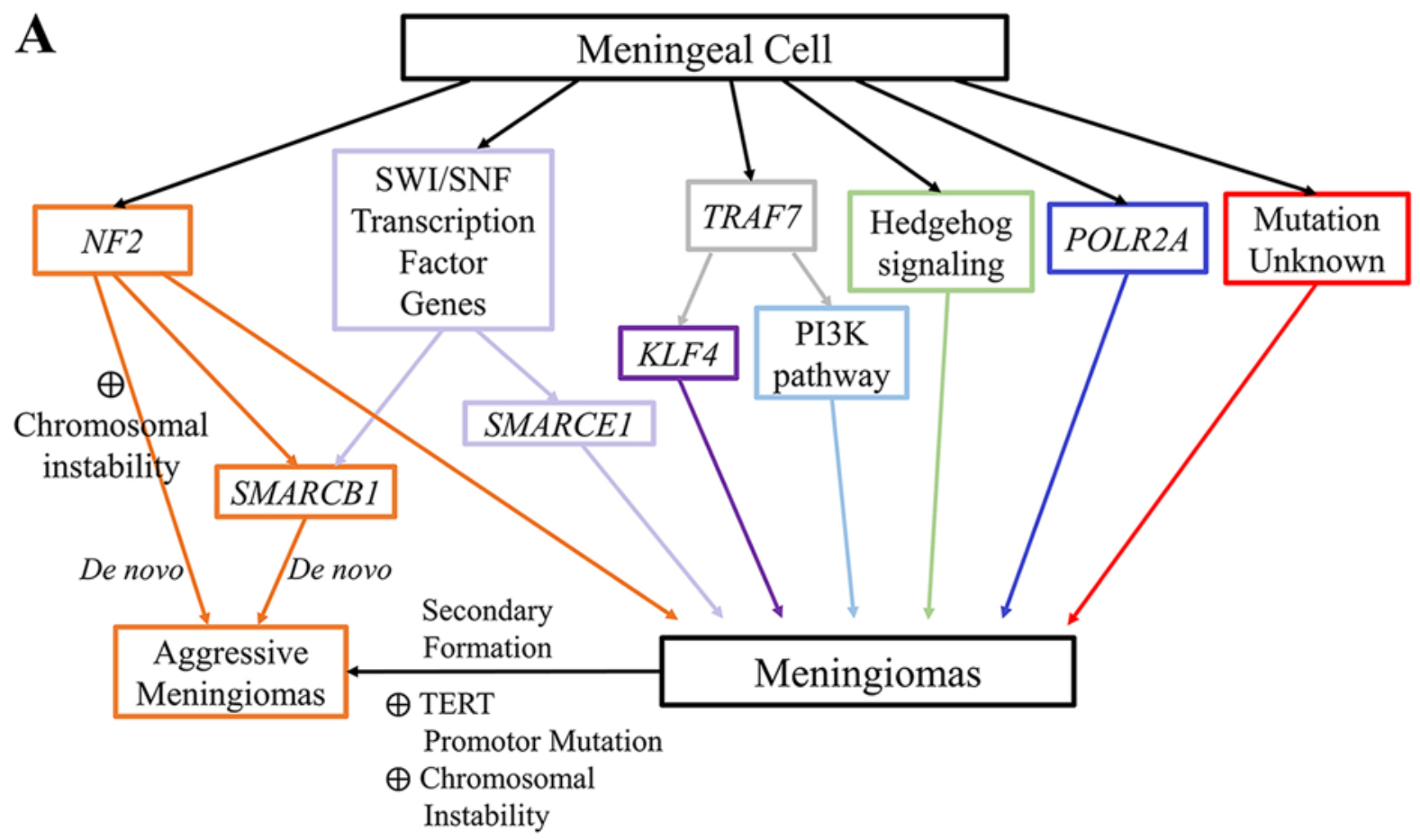

B Characteristics of the study cohort

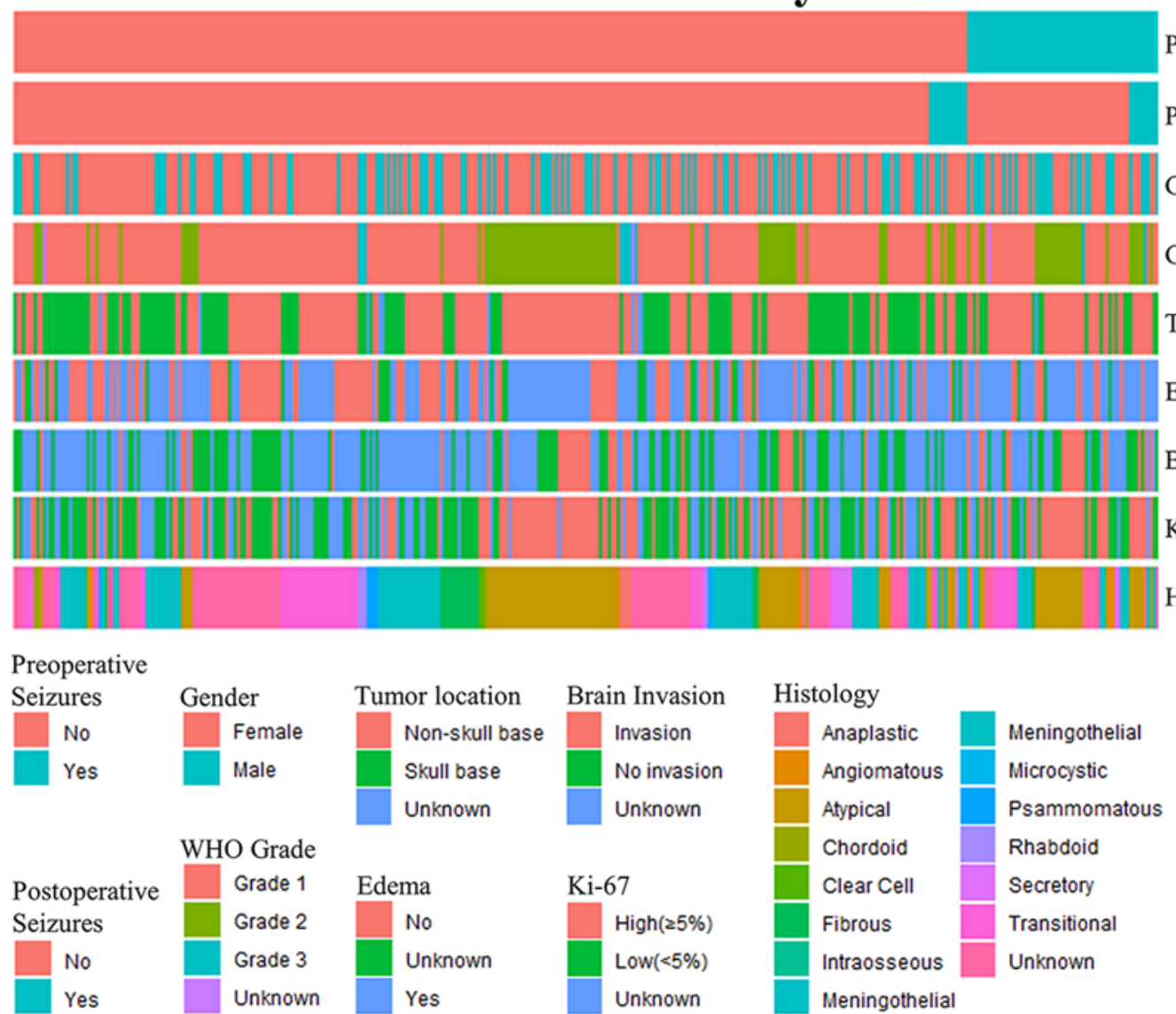

FIG. 1. A: Meningioma driver mutations and pathways. ${ }^{20,35,36}$ B: Characteristics of the study cohort. Figure is available in color online only. 
TABLE 1. AED use in patients with pre- and postoperative seizures

\begin{tabular}{lcc}
\hline \multicolumn{1}{c}{$\begin{array}{c}\text { Preop } \\
\text { AED }\end{array}$} & $\begin{array}{c}\text { Patients w/ Preop } \\
\text { Seizure }(\mathrm{n}=63)\end{array}$ & $\begin{array}{c}\text { Patients w/ Postop } \\
\text { Seizure }(\mathrm{n}=11)\end{array}$ \\
\hline Levetiracetam & $49(78 \%)$ & $7(64 \%)$ \\
\hline Valproic acid & $4(6 \%)$ & $1(9 \%)$ \\
\hline Phenytoin & $2(3 \%)$ & - \\
\hline Lacosamide & $1(2 \%)$ & - \\
\hline Lorazepam & - & $1(9 \%)$ \\
\hline Combinatorial therapy & $7(11 \%)$ & $2(18 \%)$ \\
\hline
\end{tabular}

Only patients who took AEDs preoperatively were included in this table.

whereas samples from patients without seizures had only $7.6 \%$ of their genomes altered by CNV events (Supplemental Fig. 1). In a multivariate analysis, PTBE (OR 3.11, $95 \%$ CI $1.46-6.65, \mathrm{p}=0.003)$ and atypical histology (OR $2.00,95 \%$ CI $1.03-3.90, \mathrm{p}=0.041)$ emerged as positive predictors of preoperative seizures (Fig. 3A, Supplemental Table 3). Patients with preoperative seizures also exhibited overall worse PFS (HR 2.68, 95\% CI 1.30-5.50; Fig. 3C).

\section{Seizure Formation Analyses}

In univariate analyses, NF2-mutated tumors were found to be significantly correlated with atypical histology ( $\mathrm{p}<$ $0.001)$ but not with PTBE ( $p=0.99)$; atypical histology was significantly correlated with both brain invasion $(\mathrm{p}<0.001)$ and PTBE $(\mathrm{p}<0.001)$; and brain invasion and PTBE were also significantly correlated $(\mathrm{p}<0.001)$. Mediation analysis revealed a significant indirect effect of the NF2 genomic subgroup on preoperative seizures through the sequential mediators of atypical histology and PTBE ( $p=0.012$; Fig. 4). The corresponding direct effect was not significant ( $p$ $=0.114$ ). The indirect pathway through atypical histology and brain invasion was not significant $(\mathrm{p}=0.064)$.

\section{Factors Associated With Postoperative Seizures}

The results from univariate analyses of associations between clinical or histological parameters and postoperative seizures are summarized in Table 3. Patients with postoperative seizures were more likely to present with preoperative seizures $(p=0.001)$. They were also more likely to have recurrent tumors $(\mathrm{p}=0.009)$ with atypical histology $(\mathrm{p}=0.024)$ and PTBE $(\mathrm{p}=0.020)$, which required postoperative radiation $(\mathrm{p}=0.013)$. Of the patients who received postoperative radiation, 2 underwent Gamma Knife radiosurgery while 9 received standard fractionated radiotherapy. In all cases, patients presented with postoperative seizures before receiving postoperative radiation. Notably, EOR $(p=0.830)$ and brain invasion $(p=1.000)$ were not found to be significantly correlated with postoperative seizures. For EOR in particular, there was also no observable difference between STR and GTR in the preoperative seizure patients who had seizures postoperatively ( $\mathrm{p}$ $=0.490$ ). There were no significant genomic correlations for postoperative seizures (Fig. 2B, Supplemental Table 1). However, when stratifying by tumor grade, the Hedgehog pathway-mutated tumors were found to be significantly
TABLE 2. Univariate analysis: clinical and histological correlations with preoperative seizures

\begin{tabular}{|c|c|c|c|}
\hline Variable & $\begin{array}{c}\text { No Preop } \\
\text { Seizures }\end{array}$ & $\begin{array}{c}\text { Preop } \\
\text { Seizures }\end{array}$ & $p$ Value \\
\hline \multicolumn{4}{|l|}{ Sex } \\
\hline$M$ & $96(29.3)$ & $30(45.5)$ & \multirow[t]{2}{*}{0.014} \\
\hline $\mathrm{F}$ & $232(70.7)$ & $36(54.5)$ & \\
\hline Median age in yrs (SD) & $57.6(13.1)$ & $58.2(12.2)$ & 0.430 \\
\hline Median tumor vol in $\mathrm{cm}^{3}$ (SD) & $21.3(40.0)$ & $26.6(41.6)$ & 0.471 \\
\hline \multicolumn{4}{|l|}{ WHO grade } \\
\hline 1 & $228(71.0)$ & $37(56.9)$ & \multirow[t]{2}{*}{0.029} \\
\hline$\|\&\| I \|$ & $93(29.0)$ & $28(43.1)$ & \\
\hline \multicolumn{4}{|l|}{ Tumor location } \\
\hline Skull base & $138(43.3)$ & $17(26.2)$ & \multirow[t]{2}{*}{0.012} \\
\hline Non-skull base & $181(56.7)$ & $48(73.8)$ & \\
\hline \multicolumn{4}{|l|}{ Edema } \\
\hline Present & $167(56.8)$ & $50(80.6)$ & \multirow[t]{2}{*}{$<0.001$} \\
\hline Absent & $127(43.2)$ & $12(19.4)$ & \\
\hline \multicolumn{4}{|l|}{ Brain invasion } \\
\hline Present & $35(26.5)$ & $18(50.0)$ & \multirow[t]{2}{*}{0.009} \\
\hline Absent & $97(73.5)$ & $18(50.0)$ & \\
\hline \multicolumn{4}{|l|}{ Ki-67 expression } \\
\hline Low $(<5 \%)$ & $131(53.0)$ & $18(33.9)$ & \multirow[t]{2}{*}{0.012} \\
\hline High ( $\geq 5 \%)$ & $116(47.0)$ & $37(66.1)$ & \\
\hline \multicolumn{4}{|l|}{ Histology } \\
\hline Atypical & $71(21.6)$ & $26(39.4)$ & \multirow[t]{2}{*}{0.004} \\
\hline Non-atypical & $257(78.4)$ & $40(60.6)$ & \\
\hline
\end{tabular}

Values are expressed as number (\%), unless indicated otherwise. Boldface type indicates statistical significance.

associated with postoperative seizures among WHO grade I meningiomas ( $p=0.026$; Fig. 2D, Supplemental Table 2). In a multivariate analysis, tumor recurrence (OR 2.89, 95\% CI 1.08-7.74, $\mathrm{p}=0.035)$, postoperative radiation (OR $2.82,95 \%$ CI $1.09-7.33, \mathrm{p}=0.033)$, and preoperative seizures (OR 3.54, 95\% CI 1.37-9.12, $\mathrm{p}=0.009)$ all emerged as positive predictors of postoperative seizures (Fig. 3B, Supplemental Table 4). Postoperative seizures were not significantly associated with overall worse PFS (HR 2.26, 95\% CI 0.93-5.45, $\mathrm{p}=0.063$; Fig. 3D). No patients with postoperative seizures died during this study.

\section{Discussion}

To our knowledge, this is the first study to describe significant correlations between seizures and known genomic markers in meningiomas. The occurrence of seizures at the time of presentation appears to signal a potentially more challenging clinical entity, as it is more likely to be associated with atypical histology and a corresponding somatic driver mutation in NF2, more commonly seen with aggressive meningiomas. ${ }^{19}$ Taken together, these findings suggest that preoperative seizures may portend a more challenging clinical course with an associated worse PFS.

Seizures are a relatively common presentation of me- 

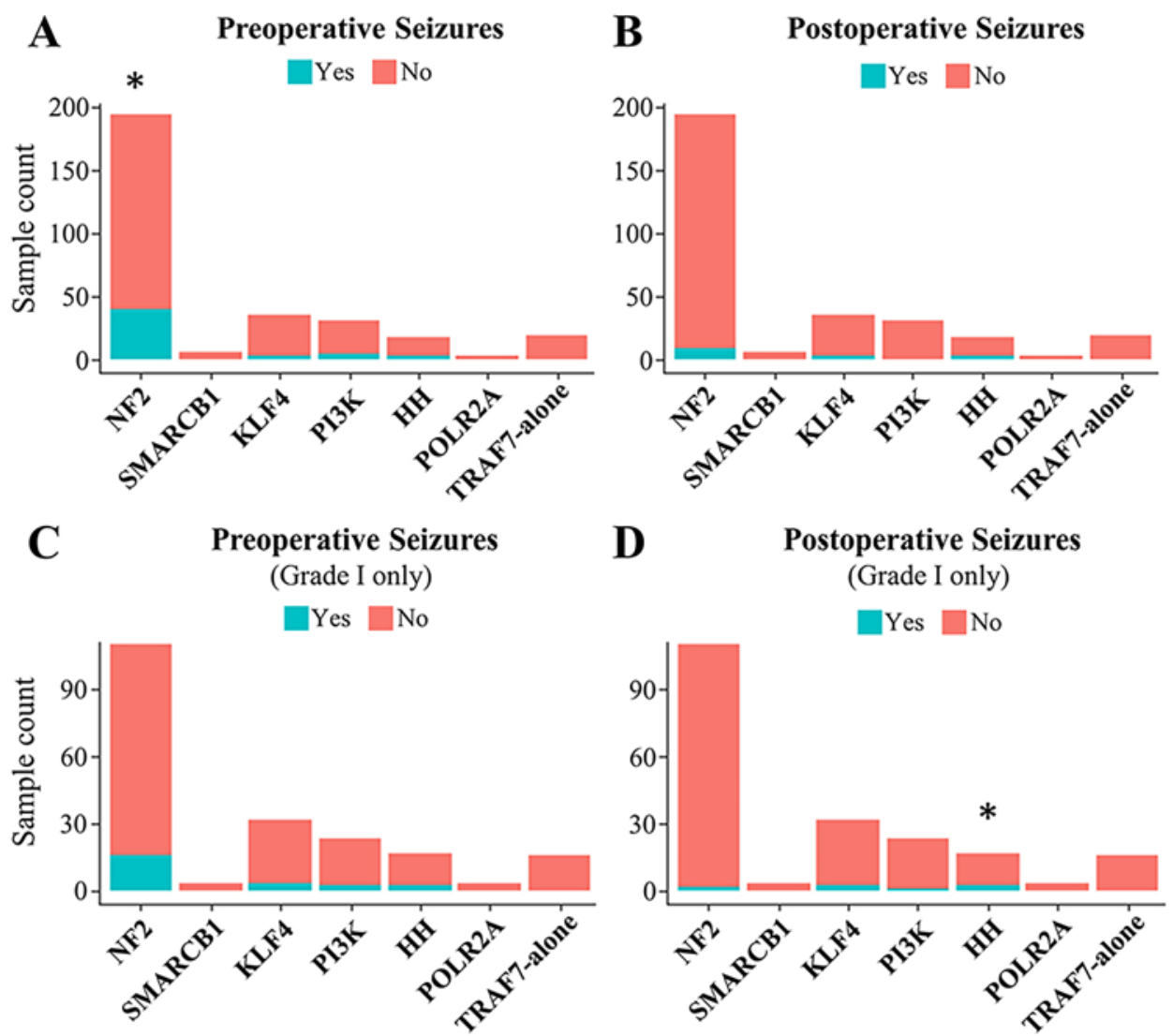

FIG. 2. Association of genomic subgroups with pre- and postoperative seizures. A: The NF2 subgroup was significantly associated with preoperative seizures. B: No significant associations between genomic subgroups and postoperative seizures were found. C: No significant associations between genomic subgroups and preoperative seizures were found among grade I tumors. D: The $\mathrm{HH}$ subgroup was significantly associated with postoperative seizures among WHO grade I tumors. Figure is available in color online only.

ningioma patients, perhaps surprisingly so given the extraaxial location of the meningiomas. Similar to others, we found that patients with preoperative seizures were more likely to have tumors with a higher grade, atypical histology, elevated Ki-67 expression, PTBE, and brain invasion. ${ }^{4,13}$ The finding that seizure patients were more likely to have tumors in the convexity fits well with the known mechanistic nature of seizures to arise from cortical irritation and corresponds nicely with our previously defined location for NF2-mutated tumors along the convexity. ${ }^{18}$

Given our previous findings and those of others, correlations between the NF2 genomic subgroup and tumorspecific characteristics suggest that NF2-mutated tumors are associated with more aggressive meningioma formation. Clark et al. was one of the first to publish findings on 7 distinct and novel genomic subgroups in meningiomas: NF2, POLR2A, SMARCB1, TRAF7, KLF4, HH, and mutation unknown. ${ }^{20}$ Harmancs et al. further explained the biological pathways underlying more aggressive atypical de novo meningioma formation and found the NF2 subgroup to be strongly correlated with these tumors. ${ }^{19}$ More recently, Youngblood et al. examined these genomic subgroups in relation to clinical correlates and found the NF2 genomic subgroup to be significantly associated with atypical histology and tumor locations in the convexity. ${ }^{18}$ In our current study, we add that patients who present with preoperative seizures are more likely to have a tumor in a non-skull base location, as well as an associated underlying somatic NF2 mutation, which is in line with these reports. While a mutation in the NF2 gene may not lead to seizure occurrence itself, its association with more aggressive characteristics may be responsible.

The underlying mechanism behind seizure formation in meningioma patients has yet to be uncovered. When Hess et al. investigated the role that brain invasion might play in the risk of seizures in meningiomas, they found correlations among PTBE, brain invasion, and preoperative seizures but concluded that the pathogenesis of PTBE in more invasive meningiomas was unclear. ${ }^{13}$ Our ability to correlate genomic data with the occurrence of seizures in meningioma patients for the first time may be an important step in understanding the underlying mechanism. We found that the NF2 genomic subgroup was not significant in the multivariate analysis for preoperative seizures, which underscores that a driver mutation is often unlikely to directly result in a clinical manifestation itself. Through mediation analysis, however, we found that the correlation between the NF2 genomic subgroup and preoperative sei- 


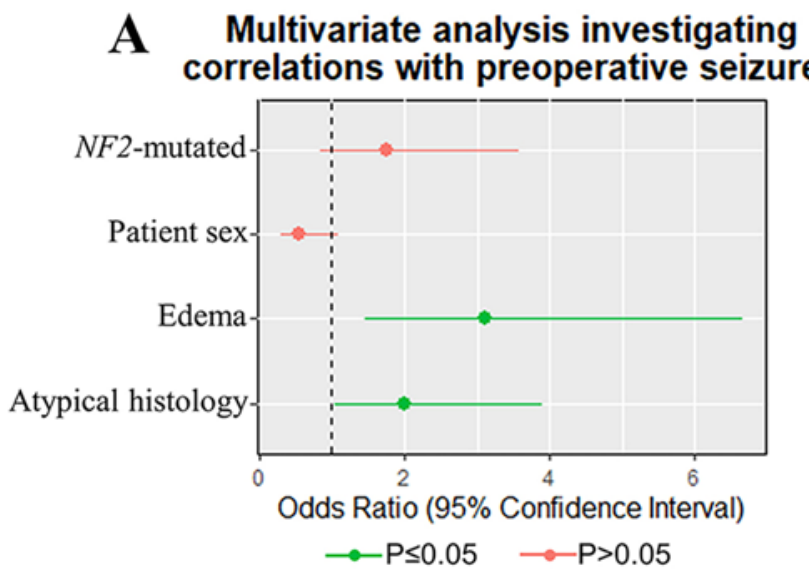

\section{C}
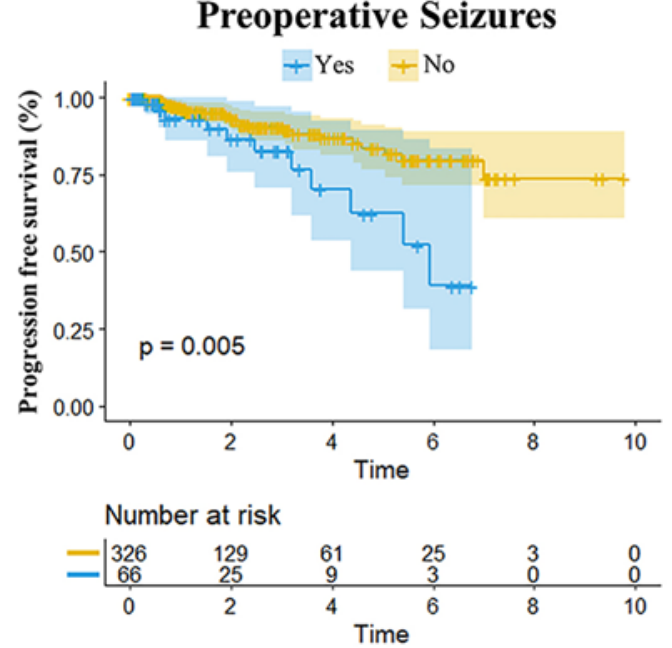

B Multivariate analysis investigating correlations with postoperative seizures

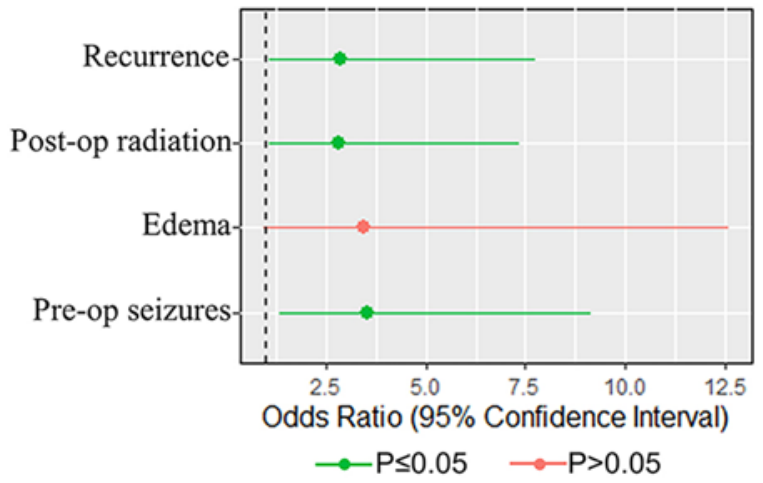

D

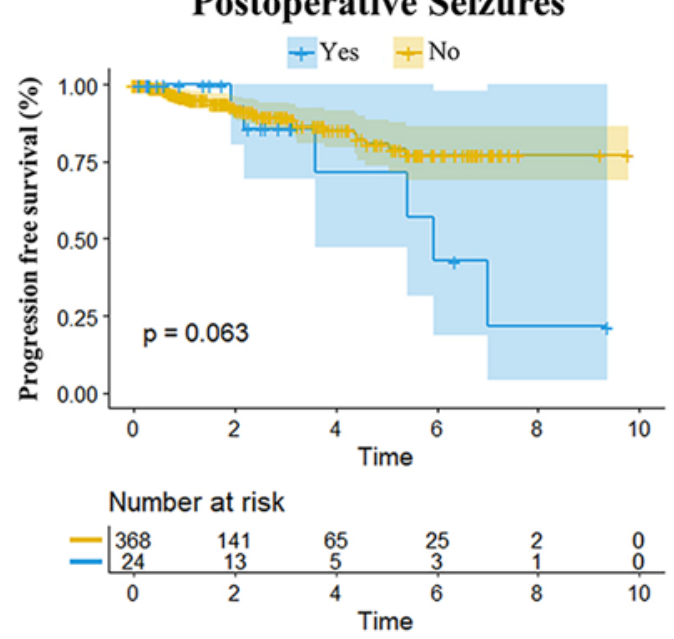

FIG. 3. Association of clinical features and NF2 status with pre- and postoperative seizures. A: Preoperative seizures were significantly associated with PTBE and atypical histology. B: Postoperative seizures were significantly associated with tumor recurrence, postoperative radiation, and preoperative seizures. C: Patients with preoperative seizures demonstrated significantly worse PFS compared to those without preoperative seizures. D: Patients with postoperative seizures did not have a significantly worse PFS compared to those without postoperative seizures. Figure is available in color online only.

zures was significantly mediated by atypical histology and PTBE in a causal pathway. An indirect pathway involving atypical histology and brain invasion was not found to be significant $(\mathrm{p}=0.064)$. Additionally, although the analysis did not reach significance $(p=0.073)$, we observed another feature associated with atypical tumors, namely an increase in the percent of genomes altered in the group with preoperative seizures, compared with that in the group without seizures ( $13.0 \%$ vs $7.6 \%$, respectively).

Other known genomic drivers, such as KLF4 mutation, have been shown to be associated with a secretory histology and subsequently with PTBE as well. ${ }^{18}$ However, our finding that the KLF4 genomic subgroup was not significant in relation to seizures leads us to posit that a more benign PTBE formation pathway may exist, different from that which occurs with more aggressive meningiomas, and one that does not lead to preoperative seizures. We hypothesize that perhaps brain invasion is the difference between these groups with regard to seizures, and further clarification is needed.
Aside from correlations with tumor genomic subgroups, the other factors we found to be associated with preoperative seizures were mostly in accordance with the literature. When Baumgarten et al. conducted a focused review on seizures in meningiomas, they described seizure patients as more likely to have PTBE and a non-skull base tumor localization..$^{21}$ Although larger tumor size was also listed as a significant risk factor, we along with others did not find an association between tumor volume and preoperative seizures. ${ }^{3,22,23}$ Interestingly, of the 20 studies Baumgarten et al. included in their systematic review, only one described tumor histological subtypes in patients with preoperative seizure. ${ }^{24} \mathrm{We}$ are therefore one of the few to report a significant association between atypical meningiomas and preoperative seizures. ${ }^{4,13}$ Furthermore, a need for future studies to examine preoperative seizures in relation to tumor histology would be useful. However, this could very well be superseded by WES and genomics data as they are more commonly used in clinical care.

When identifying risk factors associated with postoper- 


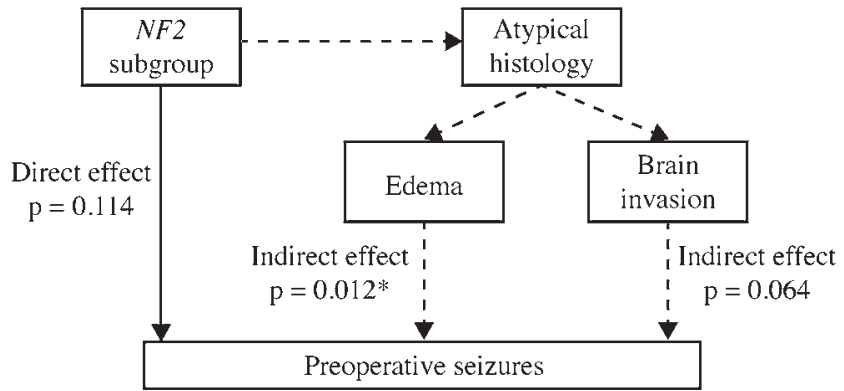

FIG. 4. Mediation analysis investigating the causal pathways between genomic subgroups and preoperative seizures. A significant indirect effect instead of a direct effect of the NF2 subgroup on preoperative seizures through the sequential mediators of atypical histology and PTBE was revealed. An indirect effect of the NF2 subgroup on preoperative seizures through the sequential mediators of atypical histology and brain invasion was suggested, but not signifiicant.

ative seizures, we found that patients were more likely to have recurrent tumors with a higher grade, atypical histology, and PTBE, which required treatment with postoperative radiation. Prior studies have shown similar results with regard to PTBE $5,25,26$ and tumor recurrence. ${ }^{4,27}$ Notably, while we along with others did not find EOR to be a significant predictor, ${ }^{3,14,24}$ Islim et al. did find a positive correlation between greater EOR and seizure freedom in patients. ${ }^{25}$ With regard to radiation, because all patients who underwent postoperative radiation had experienced postoperative seizures beforehand, it would appear that the correlation we observed has little to do with the potential of radiation to induce seizures postoperatively. Instead, our finding suggests that postoperative seizure patients are more likely to have aggressive tumor features like atypical histology, which are more typically radiated at our institution. Additionally, while our sample size was too small to discern any difference between radiation modalities, the potential for either form of radiation to be associated with postoperative seizures warrants further examination. Taken together with the finding that tumor recurrence also emerged as a predictor of postoperative seizures in the multivariate analysis, it appears that patients with postoperative seizures have a greater likelihood of experiencing an overall more complicated clinical course. Interestingly, however, the findings we observed with postoperative radiation and tumor recurrence were independent of tumor grade, which could perhaps be better explained by underlying biology.

When genomic correlations were tested within tumor grade subgroups for both pre- and postoperative seizures, analyses yielded overwhelmingly nonsignificant findings, as they were likely underpowered given the relatively small sample sizes within each tumor grade subgroup. In the case of postoperative seizures, however, Hedgehog-mutated tumors were found to be significantly associated among grade I meningiomas only. It has been well established that Hedgehog-mutated tumors more typically occur along the olfactory groove in the skull base $\mathrm{e}^{18}$ and can often be seen with bilateral frontal lobe edema. ${ }^{28}$ Unlike our finding with preoperative seizures, we did not observe a significant relationship between postoperative seizures and non-skull base
TABLE 3. Univariate analysis: clinical and histological correlations with postoperative seizures

\begin{tabular}{|c|c|c|c|}
\hline Variable & $\begin{array}{c}\text { No Postop } \\
\text { Seizures }\end{array}$ & $\begin{array}{l}\text { Postop } \\
\text { Seizures }\end{array}$ & $\mathrm{p}$ Value \\
\hline \multicolumn{4}{|l|}{ Preop seizures } \\
\hline Present & $55(14.9)$ & $11(45.8)$ & 0.001 \\
\hline Absent & $315(85.1)$ & $13(54.2)$ & \\
\hline \multicolumn{4}{|l|}{ WHO grade } \\
\hline 1 & $253(69.9)$ & $12(50.0)$ & 0.066 \\
\hline$\|\&\| \|$ & $109(30.1)$ & $12(50.0)$ & \\
\hline \multicolumn{4}{|l|}{ Tumor location } \\
\hline Skull base & $144(40.0)$ & $11(45.8)$ & 0.668 \\
\hline Non-skull base & $216(60.0)$ & $13(54.2)$ & \\
\hline \multicolumn{4}{|l|}{ Edema } \\
\hline Present & $199(59.4)$ & $18(85.7)$ & 0.020 \\
\hline Absent & $136(40.6)$ & $3(14.3)$ & \\
\hline \multicolumn{4}{|l|}{ Brain invasion } \\
\hline Present & $50(31.6)$ & $3(30.0)$ & 0.99 \\
\hline Absent & $108(68.4)$ & $7(70.0)$ & \\
\hline \multicolumn{4}{|l|}{ Histology } \\
\hline Atypical & $86(23.2)$ & $11(45.8)$ & 0.024 \\
\hline Non-atypical & $284(76.8)$ & $13(54.2)$ & \\
\hline \multicolumn{4}{|l|}{ Recurrence } \\
\hline Recurrent tumor & 54 (15.1) & $9(37.5)$ & 0.009 \\
\hline Primary tumor & $304(84.9)$ & $15(62.5)$ & \\
\hline \multicolumn{4}{|l|}{ Postop radiation } \\
\hline Yes & $67(18.1)$ & $10(41.7)$ & 0.013 \\
\hline No & $303(81.9)$ & $14(58.3)$ & \\
\hline \multicolumn{4}{|l|}{ EOR } \\
\hline STR & $158(48.3)$ & $12(52.2)$ & 0.830 \\
\hline GTR & $169(51.7)$ & $11(47.8)$ & \\
\hline
\end{tabular}

Values are expressed as number (\%), unless indicated otherwise. Boldface type indicates statistical significance.

tumor locations. Thus, the underlying molecular makeup of the tumor and perhaps the degree and effect of associated edema, rather than its location, may be more important for explaining the persistence of seizures after resection.

While AEDs are commonly used and can control most seizures in meningiomas, some seizures can be pharmacoresistant, requiring multiple agents. ${ }^{6,16}$ Indeed, approximately $11 \%$ of preoperative seizure patients in our cohort had received combinatorial AED therapy. Nonetheless, we observed a seizure freedom rate of $83.3 \%$, which is noticeably higher than what has been reported in other studies, with reported rates more often in the $60 \%-70 \%$ range. ${ }^{4,29}$ However, the majority of pre- and postoperative seizure patients (79.2\% and $95.8 \%$, respectively) were still actively taking their AED medications at the time of data collection. Through an analysis of the seizure-free patients who were able to discontinue AED use postoperatively, we found that they were more likely to have undergone a GTR. This finding lends further support to the view that a greater EOR can help alleviate associated symptoms, 
such as seizures, ${ }^{30,31}$ as well as offer the potential benefits of a decreased recurrence risk. The usefulness of perioperative AED administration, on the other hand, appears less beneficial. Although nearly half of the cohort without preoperative seizures received perioperative levetiracetam (Keppra), its use was not a protective factor against the occurrence of postoperative seizures. When considering the cost and side effects, most studies have concluded that the overall benefit of the routine application of AEDs to meningioma patients remains unclear. ${ }^{2,8,32}$

Study limitations included the retrospective nature of patient data collection and the limited data for important histological variables, primarily for evidence of brain invasion. After 2016, our neuropathologists retrospectively reviewed cases with evidence of brain invasion and changed these cases from tumor grade I to grade II. Prior to 2016, it is possible that some cases were not reevaluated accordingly. Several cases prior to 2016 also had no mention of brain invasion or instead had evidence of brain invasion marked as "unknown." Thus, comprehensive analyses of brain invasion in relation to other key variables that we hypothesized may be involved in a more aggressive PTBE formation pathway were likely underpowered. Other studies have also cited difficulties in reporting on brain invasion. ${ }^{33,34}$ When Brokinkel et al. conducted a recent systematic review on brain invasion in meningiomas, they concluded that the varying techniques for identifying brain invasion among researchers have made it difficult to consolidate findings. ${ }^{14}$ Further work to standardize the identification of brain invasion is necessary in order to better understand this key variable. Finally, relatively small sample sizes for each genomic subgroup could account for observing certain trends, but not significant results.

\section{Conclusions}

Patients with meningiomas who present with seizures may indeed have a more aggressive meningioma and tend to experience a more complicated clinical course. While patients with preoperative seizures may be more likely to have recurrent tumors, pursuing a more extensive resection may help in weaning them off of AED use postoperatively. Patients who undergo radiation and/or have a recurrence are at risk for postoperative seizures, regardless of the amount of residual tumor left after resection. Although further work is needed, our ability to correlate a mutation in the NF2 gene with the occurrence of preoperative seizures in meningioma patients may be the first step to establishing a more in-depth understanding of the relationship between seizures and meningiomas. For institutions without WES capabilities, encountering patients with meningiomas and preoperative seizures, along with a convexity location and PTBE, may signal a tumor with an underlying NF2 mutation and one that may be more likely to have characteristics of a more aggressive meningioma.

\section{Acknowledgments}

We are grateful to the patients who contributed to this study. This study was supported by the Gregory M. Kiez and Mehment Kutman Foundation. This study was also supported by the Connecticut Brain Tumor Alliance.

\section{References}

1. Englot DJ, Chang EF, Vecht CJ. Epilepsy and brain tumors. Handb Clin Neurol. 2016;134:267-285.

2. Xue H, Sveinsson O, Tomson T, Mathiesen T. Intracranial meningiomas and seizures: a review of the literature. Acta Neurochir (Wien). 2015;157(9):1541-1548.

3. Chaichana KL, Pendleton C, Zaidi H, et al. Seizure control for patients undergoing meningioma surgery. World Neurosurg. 2013;79(3-4):515-524.

4. Chozick BS, Reinert SE, Greenblatt SH. Incidence of seizures after surgery for supratentorial meningiomas: a modern analysis. J Neurosurg. 1996;84(3):382-386.

5. Lieu AS, Howng SL. Intracranial meningiomas and epilepsy: incidence, prognosis and influencing factors. Epilepsy Res. 2000;38(1):45-52.

6. Taphoorn MJ, Klein M. Cognitive deficits in adult patients with brain tumours. Lancet Neurol. 2004;3(3):159-168.

7. van Breemen MS, Wilms EB, Vecht CJ. Epilepsy in patients with brain tumours: epidemiology, mechanisms, and management. Lancet Neurol. 2007;6(5):421-430.

8. Wirsching HG, Morel C, Gmür C, et al. Predicting outcome of epilepsy after meningioma resection. Neuro Oncol. 2016; 18(7):1002-1010.

9. DeAngelis LM. Brain tumors. N Engl J Med. 2001;344(2): 114-123.

10. Kargiotis O, Markoula S, Kyritsis AP. Epilepsy in the cancer patient. Cancer Chemother Pharmacol. 2011;67(3):489-501.

11. Luyken C, Blümcke I, Fimmers R, et al. The spectrum of long-term epilepsy-associated tumors: long-term seizure and tumor outcome and neurosurgical aspects. Epilepsia. 2003; 44(6):822-830.

12. Sirven JI, Wingerchuk DM, Drazkowski JF, et al. Seizure prophylaxis in patients with brain tumors: a meta-analysis. Mayo Clin Proc. 2004;79(12):1489-1494.

13. Hess K, Spille DC, Adeli A, et al. Brain invasion and the risk of seizures in patients with meningioma. J Neurosurg. 2018; 130(3):789-796.

14. Brokinkel B, Hess K, Mawrin C. Brain invasion in meningiomas-clinical considerations and impact of neuropathological evaluation: a systematic review. Neuro Oncol.2017;19(10): 1298-1307.

15. Stockhammer F, Misch M, Helms HJ, et al. IDH1/2 mutations in WHO grade II astrocytomas associated with localization and seizure as the initial symptom. Seizure. 2012; 21(3):194-197.

16. Vecht CJ, Kerkhof M, Duran-Pena A. Seizure prognosis in brain tumors: new insights and evidence-based management. Oncologist. 2014;19(7):751-759.

17. Takahashi JA, Ueba T, Hashimoto N, et al. The combination of mitotic and Ki-67 indices as a useful method for predicting short-term recurrence of meningiomas. Surg Neurol. 2004;61(2):149-156.

18. Youngblood MW, Duran D, Montejo JD, et al. Correlations between genomic subgroup and clinical features in a cohort of more than 3000 meningiomas. J Neurosurg. 2020;133(5): $1345-1354$

19. Harmancı AS, Youngblood MW, Clark VE, et al. Integrated genomic analyses of de novo pathways underlying atypical meningiomas. Nat Commun. 2017;8:14433.

20. Clark VE, Erson-Omay EZ, Serin A, et al. Genomic analysis of non-NF2 meningiomas reveals mutations in TRAF7, KLF4, AKT1, and SMO. Science. 2013;339(6123):1077-1080.

21. Baumgarten P, Sarlak M, Baumgarten G, et al. Focused review on seizures caused by meningiomas. Epilepsy Behav. 2018;88:146-151.

22. Islim AI, Mohan M, Moon RDC, et al. Incidental intracranial meningiomas: a systematic review and meta-analysis of prognostic factors and outcomes. J Neurooncol. 2019;142(2): 211-221. 
23. Kawaguchi T, Kameyama S, Tanaka R. Peritumoral edema and seizure in patients with cerebral convexity and parasagittal meningiomas. Neurol Med Chir (Tokyo). 1996;36(8): 568-574.

24. Skardelly M, Rother C, Noell S, et al. Risk factors of preoperative and early postoperative seizures in patients with meningioma: a retrospective single-center cohort study. World Neurosurg. 2017;97:538-546.

25. Islim AI, Ali A, Bagchi A, et al. Postoperative seizures in meningioma patients: improving patient selection for antiepileptic drug therapy. J Neurooncol. 2018;140(1):123-134.

26. Zhang BO, Wang D, Guo Y, Yu J. Clinical multifactorial analysis of early postoperative seizures in elderly patients following meningioma resection. Mol Clin Oncol. 2015;3(3): 501-505.

27. Das RR, Artsy E, Hurwitz S, et al. Outcomes after discontinuation of antiepileptic drugs after surgery in patients with low grade brain tumors and meningiomas. J Neurooncol. 2012;107(3):565-570.

28. Boetto J, Bielle F, Sanson M, et al. SMO mutation status defines a distinct and frequent molecular subgroup in olfactory groove meningiomas. Neuro Oncol. 2017;19(3):345-351.

29. Zheng Z, Chen P, Fu W, et al. Early and late postoperative seizure outcome in 97 patients with supratentorial meningioma and preoperative seizures: a retrospective study. $J$ Neurooncol. 2013;114(1):101-109.

30. Nanda A, Bir SC, Maiti TK, et al. Relevance of Simpson grading system and recurrence-free survival after surgery for World Health Organization Grade I meningioma. J Neurosurg. 2017;126(1):201-211.

31. Gousias K, Schramm J, Simon M. The Simpson grading revisited: aggressive surgery and its place in modern meningioma management. J Neurosurg. 2016;125(3):551-560.

32. Sughrue ME, Rutkowski MJ, Chang EF, et al. Postoperative seizures following the resection of convexity meningiomas: are prophylactic anticonvulsants indicated? Clinical article. $J$ Neurosurg. 2011;114(3):705-709.

33. Spille DC, Heß K, Sauerland C, et al. Brain invasion in meningiomas: incidence and correlations with clinical variables and prognosis. World Neurosurg. 2016;93:346-354.

34. Backer-Grøndahl T, Moen BH, Arnli MB, et al. Immunohistochemical characterization of brain-invasive meningiomas. Int J Clin Exp Pathol. 2014;7(10):7206-7219.
35. Clark VE, Harmancı AS, Bai H, et al. Recurrent somatic mutations in POLR2A define a distinct subset of meningiomas. Nat Genet. 2016;48(10):1253-1259.

36. Smith MJ, O'Sullivan J, Bhaskar SS, et al. Loss-of-function mutations in SMARCE1 cause an inherited disorder of multiple spinal meningiomas. Nat Genet. 2013;45(3):295-298.

\section{Disclosures}

The authors report no conflict of interest concerning the materials or methods used in this study or the findings specified in this paper.

\section{Author Contributions}

Conception and design: Moliterno, Gupte, Li. Acquisition of data: Moliterno, Gupte, Yalcin, Youngblood, Miyagishima, Mishra-Gorur, Zhao, Huttner, McGuone, Blondin, Contessa, Fulbright, Erson-Omay. Analysis and interpretation of data: Moliterno, Gupte, Li, Jin, Yalcin, Erson-Omay. Drafting the article: Moliterno, Gupte, Li, Jin, Yalcin, Erson-Omay. Critically revising the article: Moliterno, Gupte, Li, Jin, Yalcin, Miyagishima, Mishra-Gorur, Zhao, Antonios, Gunel, Erson-Omay. Reviewed submitted version of manuscript: all authors. Approved the final version of the manuscript on behalf of all authors: Moliterno. Statistical analysis: Gupte, Li, Jin, Yalcin, Erson-Omay.

\section{Supplemental Information}

Online-Only Content

Supplemental material is available with the online version of the article.

Supplemental Tables and Fig. https://thejns.org/doi/suppl/ 10.3171/2020.7.JNS201042.

\section{Correspondence}

Jennifer Moliterno: Yale School of Medicine, New Haven, CT. jennifer.moliternogunel@yale.edu. 